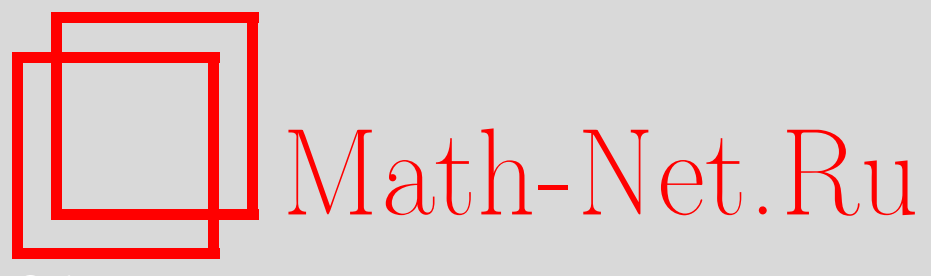

Ю. А. Брудный, М. Г. Зайденберг, А. Л. Колдобский, В. Я. Лин, Б. С. Митягин, С. Норвидас, Е. М. Семенов, П. В. Семенов, Евгений Алексеевич Горин (некролог), УМH, 2019, том 74, выпуск 5, 170-180

DOI: https://doi.org/10.4213/rm9874

Использование Общероссийского математического портала Math-Net.Ru подразумевает, что вы прочитали и согласны с пользовательским соглашением http: //www.mathnet.ru/rus/agreement

Параметры загрузки:

IP: 54.224 .135 .184

26 апреля 2023 г., 17:48:49

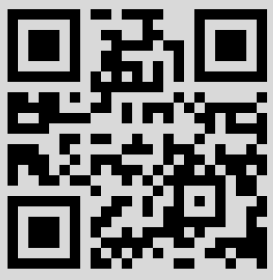




\section{Евгений Алексеевич Горин}

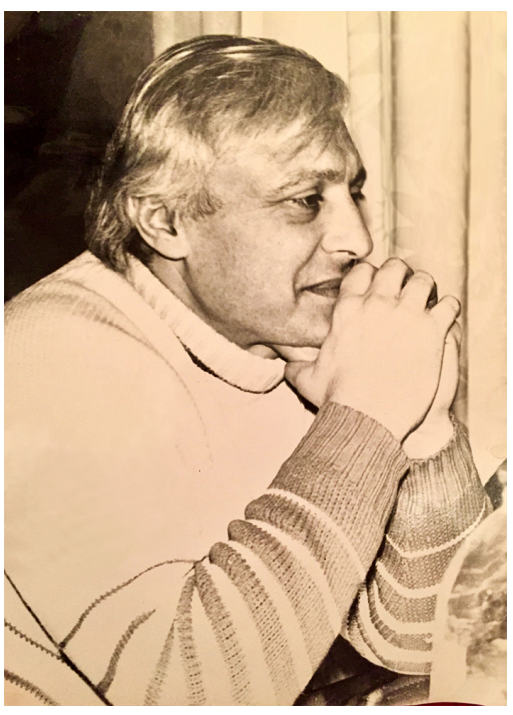

4 октября 2018 г. скончался замечательный отечественный математик Евгений Алексеевич Горин.

Е. А. Горин родился 28 января 1936 г. в Москве. Его отец, Алексей Федорович Горин (1903-1953) был уроженцем деревни Верхнее Хорошово Коломенского уезда (и старшим сыном в бедной крестьянской семье, где было 11 детей); он начал работать у станка в 13 лет, был слесарем высшего разряда, окончил МВТУ им. Н.Э. Баумана. На пике своей деятельности он был известным московским инженером, директором ряда крупных московских заводов, а в годы Великой Отечественной войны - директором важного оборонного Тормозного завода.

Мать, Лидия Семеновна Горина (1912-1991), родилась в г. Николаеве, вышла замуж, когда была работницей Тормозного завода. Перед войной, имея двоих детей, окончила Московский государственный педагогический институт (ныне МПГУ) по специальности "русский язык и литература", долгое время была заведующей учебной частью на курсах повышения квалификации учителей.

Со своей будущей женой Ириной Александровной Красовской Евгений Алексеевич познакомился на мехмате МГУ им. М.В. Ломоносова; она училась на курс младше его. Впоследствии Ирина Александровна многие годы проработала доцентом кафедры прикладной математики Московского инженерно-строительного института (ныне университета). Почти шестьдесят лет она была бессменной подругой и спутницей Евгения Алексеевича. Их сын Андрей окончил МИЭМ; по специальности он программист. Ныне он крупный предприниматель. У Евгения Алексеевича и Ирины Александровны трое внуков; их правнук родился незадолго до кончины Евгения Алексеевича.

Е. А. Горин учился в московской школе № 167 (ныне № 2054) в Дегтярном переулке; интерес и любовь к математике привила ему замечательная учительница Эльфрида Моисеевна Абезгаус. В школьные годы Евгений успешно выступал на Московской математической олимпиаде школьников; в 1953 г., сразу по окончании школы, поступил в МГУ.

Набор на мехмат 1953 г. - первый, который с первого курса обучался в новом, только что возведенном на Ленгорах, Главном здании МГУ. Это был первый набор в практически современном объеме приема на первый курс (450 мест), на него

DOI: https://doi.org/10.4213/rm9874 
поступали абитуриенты сразу двух школьных выпусков. По мнению многих, в истории мехмата этот набор был одним из самых "сильных", состоял из необыкновенно талантливых людей. Из его рядов вышло немало известных профессоров мехмата, сотрудников Математического института им. В. А. Стеклова, несколько всемирно известных математиков, директоров институтов, видных экономистов, программистов, лауреатов госпремий.

Но, пожалуй, основное - это был первый курс мехмата, набранный в год, когда не стало “лучшего друга всех физкультурников". Это поколение, студенческие годы которого пришлись на самое начало времени, позже названного оттепелъю, когда в жизни страны “. . . неуклюже зашевелился грузный лед”. А.Н.Колмогоров говорил об этом времени так: "Главное было то, что в 1953 году появилась надежда". Двадцатилетие Евгения Алексеевича практически совпало с датой проведения в 1956 г. $\mathrm{XX}$ съезда КПСС, положившего начало фундаментальным изменениям тогдашнего социума; например, понятие враг народа официально было изъято из юридических нормативов в 1958 г., в год, когда Е. А. Горин окончил МГУ.

Студенческие годы в заметной степени сформировали стиль Евгения Алексеевича во взаимоотношениях, отличительными чертами которого на протяжении всей его жизни стали свобода и открытость, теплота и дружелюбность.

Становление Е.А. Горина как видного математика пришлось, пожалуй, на 15 лет после окончания мехмата. В это время была окончена аспирантура, пройден путь от ассистента до старшего научного сотрудника кафедры теории функций и функционального анализа МГУ, написаны существенные научные работы, защищены кандидатская и докторская диссертации. В год своего тридцатилетия (1966 г.) Евгений Алексеевич выступал на Международном конгрессе математиков.

Вообще, время с 1958 по 1973 г. (год смерти И. Г. Петровского) теперь часто называют золотыми годами московской математики, и вклад Евгения Алексеевича в справедливость этой оценки несомненен. Его многолетний научный семинар "Банаховы алгебры и комплексный анализ" (совместный с В. Я. Лином) стал одной из явных точек накопления в общей яркой палитре отечественной математики тех лет. Многие из участников этого семинара, действовавшего с 1964 по 1987 г., сформировались в его рамках как профессиональные математики, стали профессорами российских и зарубежных университетов. Всего под руководством Е. А. Горина защищено 28 кандидатских диссертаций.

K этому же времени следует отнести и начало огромной работы, проделанной Евгением Алексеевичем в издательстве "Мир" в качестве редактора и переводчика. Благодаря его высокопрофессиональному и самоотверженному труду поколения советских математиков познакомились с рядом выдающихся монографий и учебников, среди которых "Лекции о теоремах Шоке" Р. Фелпса, "Методы гильбертова пространства" K. Морена, "Топологические векторные пространства" Х. Шефера, "Банаховы пространства аналитических функций" К. Гофмана, "Равномерные алгебры" Т. Гамелина, “Функциональный анализ” У. Рудина. Значение этой работы для математического сообщества невозможно переоценить.

Заряд социального оптимизма оттепельных лет заметно убывал в семидесятые годы, а к середине восьмидесятых, пожалуй, стал и вовсе иссякать. Резко менялись и общая социальная атмосфера, и ситуация в образовании и науке, и положение дел на мехмате МГУ. Своего рода отдушиной для Евгения Алексеевича стали ежегодные Воронежские зимние математические школы, в организации и проведении которых он принимал самое действенное участие и которые, начиная примерно с 1970 г., стали одной из основных точек притяжения для сотен отечественных математиков от Бреста 
до Чукотки. Евгений Алексеевич руководил работой одной из самых загруженных секций Воронежских ЗМШ - секции функционального анализа, на которой делалось около 40 докладов и сообщений. Так сложилось, что на время проведения Воронежских ЗМШ приходился и день рождения Евгения Алексеевича; это был праздник всей школы.

На мехмате МГУ Е.А. Горин работал почти четверть века, из них 15 лет со степенью доктора наук, в должности старшего научного сотрудника. В 1987 г., вскоре после пятидесятилетия, Евгений Алексеевич принял сложное для себя решение: перейти в Московский государственный педагогический институт (ныне МПГУ) на должность профессора кафедры математического анализа. Разрыв с МГУ, с Alma Mater, был болезненным для него. Впрочем, как писал несколько по иному поводу Ю. Трифонов, “...это были уже совсем иные времена, и даже не те, что наступили потом, а совсем, совсем иные времена...": заканчивалось ускорение, начинала громыхать перестройка, вновь возникали надежды и иллюзии... .

Несмотря на все сложности, Евгений Алексеевич успешно продолжал свою математическую деятельность, вплоть до выхода в 2016 г. на пенсию. Им были получены значительные результаты в различных разделах функционального анализа, см. [23], [24], [26], [28]-[31], [35] и детальнее ниже. В МПГУ он вел активную научную и научно-методическую работу, руководил работой аспирантов, в 2011 г. был отмечен премией им. П. С. Новикова.

На протяжении многих лет и до самого последнего времени Евгений Алексеевич был членом редколлегии (редсовета) журналов "Функциональный анализ и его приложения" и "Russian Journal of Mathematical Physics". В круг его ответственности входил широкий спектр направлений, от теории меры и геометрии банаховых пространств до комплексного анализа и теории чисел. Е. А. Горин являлся членом Московского и Американского математических обществ, многократно выезжал за рубеж с лекциями и докладами.

Научной работой Евгений Алексеевич начал заниматься сначала у А. Б. Шидловского, затем у Ю.М. Смирнова, под руководством которого им был получен первый научный результат. Ю. М. Смирнов спрашивал, существует ли равномерно гомеоморфное вложение произвольного метрического пространства со счетной базой в гильбертово пространство. В работе [2] Е. А. Горин показал, что $n$-мерное эвклидово пространство равномерно гомеоморфно ограниченному подмножеству в $L_{2}$. Лишь в 1969 г. П. Энфло дал отрицательный ответ на вопрос Ю. М. Смирнова. Оказалось, что сепарабельное банахово пространство $c_{0}$ не может быть равномерно гомеоморфно вложено в $L_{2}$. Затем в 1985 г. И. Ахарони, Б. Морри и Б. С. Митягин доказали, что тем же свойством обладают все пространства $l_{p}$ при $2<p<\infty$ и что для любого $p \in[1,2]$ существует равномерно гомеоморфное вложение $l_{p}$ в $L_{2}$. Так сложилось, что все же первой публикацией Евгения Алексеевича стала его совместная с Б. С. Митягиным работа [1] о системах норм, которая позже, к 1970 г., оказалась полезной и существенной в решении серии вопросов о базисах в пространствах аналитических функций (В. П. Захарюта) и об эквивалентности базисов в гильбертовых шкалах (Б. С. Митягин).

Позже Евгений Алексеевич стал заниматься функциональным анализом и дифференциальными уравнениями, много лет был активным участником семинара И. М. Гельфанда. Научным руководителем Е.А. Горина на старших курсах и в аспирантуре мехмата был Г.Е. Шилов. По его совету Евгений Алексеевич написал одну из своих наиболее цитируемых работ - "Об асимптотических свойствах многочленов и алгебраических функций от нескольких переменных" [3]. Он показал, что теория 
Тарского-Зайденберга о сохранении полуалгебраичности множеств при полиномиальных отображениях может быть с успехом использована в теории дифференциальных уравнений в частных производных. Теория Тарского-Зайденберга позволяла не только устанавливать гладкость, но и предъявлять эффективные оценки роста решения при росте порядка производной (для аналитичности в случае одной переменной по порядку получается $n$ !, а для уравнения теплопроводности по временной переменной будет $\left.(n !)^{2}\right)$. Другим замечательным результатом, полученным в статье [3], было описание уравнений, для которых гипоэллиптичность имеет место лишь по части переменных. Ссылки на эту статью можно найти в работах Л. Хёрмандера, В. И. Арнольда и многих других. Кстати, она была первой из написанных студентами мехматского выпуска 1958 г. для основного раздела УМН.

В одной из первых своих работ [8] Е. А. Горин дал алгебраическое описание тех уравнений в частных производных с постоянными коэффициентами, для которых задача Коши разрешима в лебеговых $L_{2}$-пространствах для всех начальных данных из тех же пространств. Типичным примером такого сорта является уравнение распространения звука в вязком газе. Для систем уравнений возникали дополнительные препятствия. Тем не менее в дальнейшем Е.А. Горин нашел широкие классы систем, для которых разрешимость задачи Коши сохраняется. Эти работы вошли в его кандидатскую диссертацию "Частично гипоэллиптические дифференциальные уравнения в частных производных с постоянными коэффициентами” (1962 г.).

Впоследствии занятия дифференциальными уравнениями продолжались в совместных работах с В. В. Грушиным [5], [7], [9]. В частности, в них было доказано, что у всех уравнений, кроме гипоэллиптических, множество особенностей не компактно. Кроме того, было показано, что есть уравнения, все решения которых приобретают дополнительную гладкость при дифференцировании по выделенному переменному. Например, в размерности $\geqslant 2$ таким является уравнение

$$
\frac{\partial}{\partial x_{1}} \Delta u=u
$$

где $\Delta$ - оператор Лапласа. Сопоставление таких фактов затем позволило в совместной статье по-новому взглянуть на проблему гипоэллиптичности в целом.

В годы аспирантуры Е. А. Горин успешно занимался также теорией банаховых алгебр. После защиты кандидатской диссертации эта тематика стала для него одной из ключевых. В первой публикации этого периода [6] было снято предположение о симметричности в обобщении классической теоремы Стоуна-Вейерштрасса, предложенном И. Кацнельсоном; достаточным оказалось требование ограниченности идемпотентов в факторкольцах коммутативной полупростой нормированной алгебры. Впоследствии Е.А. Горин много внимания уделял проблемам равномерной аппроксимации. Совместно со своим аспирантом М. И. Караханяном, Евгений Алексеевич нашел обобщения в рамках банаховых алгебр одной аппроксимационной теоремы Е. М. Чирки, а затем, вместе с М.С. Мельниковым и М.И. Караханяном, предложил новые методы построения экзотических равномерных алгебр (скажем, аналитических алгебр, в которых все точки пространства максимальных идеалов суть точки пика). Им построены теория относительно максимальных подалгебр, конструкции нелокальных производных и нелокальных алгебр, получены далеко идущие обобщения теоремы Фуглида-Путнама о коммутаторах, охарактеризованы алгебры с биголоморфно однородными шарами, описаны подалгебры конечной коразмерности. В заметке [18] Е. А. Горин построил первый пример алгебры, в которой группа всех обратимых элементов не разделяется характерами, тем самым показав непродолжаемость классического результата теории Гельфанда о разделимости характерами групп обратимых элементов в полупростых коммутативных банаховых алгебрах. 
В 1972 г. Е. А. Горин защитил в МГУ докторскую диссертацию "Некоторые вопросы теории коммутативных банаховых алгебр и гармонического анализа" (оппоненты: Д. К. Фаддеев, Д. А. Райков, В. И. Арнольд). Ее основу составили, пожалуй, наиболее известные в математическом сообществе работы Е. А. Горина (частью совместные с В.Я. Лином) по алгебраическим уравнениям с непрерывными или голоморфными коэффициентами и их связям с алгебраической теорией кос.

Любопытна история развития этой тематики. Она хорошо иллюстрирует математическую атмосферу тех лет на мехмате МГУ. Далее мы следуем изложению этой истории самим Евгением Алексеевичем. Все началось с предложения Б. М. Левитана заслушать на семинаре Е. А. Горина и В. Я. Лина работу В. В. Жикова об обобщении теории Бора-Фландерса, которые рассматривали уравнение

$$
\lambda^{n}+a_{1} \lambda^{n-1}+a_{2} \lambda^{n-2}+\cdots+a_{n}=0,
$$

где все коэффициенты $a_{m}$ суть почти периодические (по Бору) функции на вещественной оси $\mathbb{R}$ и, кроме того, $|d| \geqslant$ const $>0$, где $d$ - дискриминант.

Уравнение (1) имеет $n$ непрерывных решений $\lambda_{k}$, попарно различных в каждой точке, и, как установили Х. Бор и Д. Фландерс в обширной статье 1930-х годов, все эти корни почти периодичны. Нетривиальность результата объясняется тем, что даже уравнение $\lambda^{2}-a=0$ с почти периодическим $a$, вообще говоря, не имеет почти периодических решений, если у а есть нули "на бесконечности" (существованию непрерывных решений на оси это не мешает).

Было также известно, что алгебра $\mathrm{AP}(\mathbb{R})$ почти периодических функций Бора на вещественной оси изоморфна алгебре всех непрерывных функций $C(X)$ на компакте Бора $X$. Напомним, что $X$ - это связная компактная группа, двойственная к вещественной оси $\mathbb{R}_{\mathrm{d}}$, взятой в дискретной топологии. Из теорем Брушлинского-Эйленберга, Бохнера и Бора-ван Кампена следуют изоморфизмы

$$
A^{-1} / \exp A=H^{1}(X, \mathbb{Z})=\widehat{X}=\mathbb{R}_{\mathrm{d}}
$$

Здесь $A^{-1}$ - группа обратимых элементов алгебры $A=C(X), H^{1}(X, \mathbb{Z})$ - группа целочисленных чеховских когомологий и $\widehat{X}$ - группа одномерных характеров. Используя одну теорему А. Картана и теорему Аренса-Ройдена, первое из равенств (2) можно распространить на произвольные коммутативные банаховы алгебры, если вместо $X$ подставить пространство максимальных идеалов; второе же сохраняется без предположения коммутативности.

Из формулы (2) сразу вытекает разрешимость двучленных уравнений $\lambda^{n}-a=0$ в $C(X)$ для $X$ с делимой группой когомологий и обратимым $a$. Далее, аппроксимируя группу $X$ торами, Е.А. Горин и В.Я. Лин обнаружили, что разрешимы и все уравнения с $|d| \geqslant$ const $>0$ типа (1) на $C(X)$, упростив тем самым теорию Бора-Фландерса. Более того, вскоре выяснилось, что и коммутативность группы $X$ не важна.

Заменив в уравнении (1) коэффициенты $a_{m}$ на независимые комплексные переменные $z_{1}, z_{2}, \ldots, z_{n}$, можно рассматривать не уравнение, а полиномы, стоящие слева. Тогда $d=d(z)$, где $z$ - точка в $\mathbb{C}^{n}$, а $d(z)$ - полином. Пусть

$$
G_{n}=\left\{z \in \mathbb{C}^{n} \mid d(z) \neq 0\right\}
$$

После обсуждения на семинаре В. И. Арнольда Г. М. Хенкин сообщил о том, что группа кос Артина $B(n)$ изоморфна фундаментальной группе пространства $G_{n}$ полиномов степени $n$ с простыми корнями, а В. И. Арнольд подробно рассказал Е. А. Горину 
и В.Я. Лину о группе кос и дал ссылки на важные работы, с ней связанные. Напомним, что группа $B(n)$ допускает систему образующих $\sigma_{1}, \sigma_{2}, \ldots, \sigma_{n-1}$, связанных соотношениями

$$
\sigma_{i+1} \sigma_{i} \sigma_{i+1}=\sigma_{i} \sigma_{i+1} \sigma_{i}, \quad \sigma_{i} \sigma_{k}=\sigma_{k} \sigma_{i} \quad \text { при }|i-k| \geqslant 2 .
$$

Из сказанного выше следует, что приводимость всех полиномов степени $\leqslant n$ с обратимым дискриминантом над $C(X)$, где $X$ - конечный комплекс, равносильна тривиальности группы гомоморфизмов из $\pi_{1}(X)$ в $B(n)$. В то же время приводимость двучленных полиномов получается, если $B(n)$ заменить на $\mathbb{Z}$. Так как группа кос не имеет элементов конечного порядка, то второе условие, вообще говоря, слабее первого.

Легко показать, что

$$
B(n) / B^{\prime}(n)=\mathbb{Z},
$$

где $B^{\prime}(n)$ - коммутант группы кос (порожденный элементами вида $\sigma_{i} \sigma_{i}^{-1}$ ). Коммутант является фундаментальной группой многообразия $\left\{z \in \mathbb{C}^{n} \mid d(z)=1\right\}$. Поэтому решение проблемы требовало выяснения свойств группы $B^{\prime}(n)$.

В работе [12] было найдено конечное копредставление группы $B^{\prime}(n)$, т. е. эта группа была предъявлена как порожденная конечным семейством образующих и соотношений. Из этого копредставления вытекало, что $B^{\prime}(3)$ - свободная группа ранга 2 , группа $B^{\prime}(4)$ - полупрямое произведение двух свободных групп ранга 2 и $B^{\prime \prime}(n)=B^{\prime}(n)$ при $n \geqslant 5$.

Много лет спустя Е. А. Горин при $n \geqslant 4$ вывел соотношение

$$
\sigma_{1}^{-1} \sigma_{3}=\sigma_{2}^{-1} \sigma_{1}^{-1} \cdot\left[\sigma_{1}^{-1} \sigma_{3}, \sigma_{1} \sigma_{2}^{-1}\right] \cdot \sigma_{1} \sigma_{2},
$$

которое немедленно приводит к совпадению коммутантов $B^{\prime \prime}(n)=B^{\prime}(n)$ при $n \geqslant 5$. Конечно, упомянутое выше копредставление дает гораздо больше.

В конце 1970-х годов Евгений Алексеевич заинтересовался неравенствами Бернштейна в контексте спектральной теории. В 1968-1971 гг. Ф. Бонсалл и М. Крабб, А. Браудер, В.Э. Кацнельсон и А. М. Синклер независимо обнаружили, что для любого эрмитова элемента $a \in A$, т. е. элемента $a$ комплексной банаховой алгебры, для которого $\|\exp (i t a)\|=1$ при всех $t \in \mathbb{R}$, имеет место равенство

$$
\|a\|=|a|_{A},
$$

где $|a|_{A}$ - спектральный радиус. Доказательства Браудера и Кацнельсона опирались на классическое неравенство Бернштейна

$$
\left\|f^{\prime}\right\|_{\infty} \leqslant \sigma\|f\|_{\infty} \quad \forall f \in B_{\sigma},
$$

где $B_{\sigma}$ - пространство Бернштейна целых функций экспоненциального типа $\leqslant \sigma$, ограниченных на вещественной оси, а $\|f\|_{\infty}$ - равномерная норма сужения $f$ на вещественную ось. Отметим, что неравенство Бернштейна можно трактовать как равенство (5) для эрмитова оператора $a=-i d / d x$, действующего в пространстве $B_{\sigma}$; действительно, в этом случае $\operatorname{spec}(a)=[-\sigma, \sigma]$.

В 1977 г. появилась работа Х. Кёнига, в которой исследовался вопрос о том, когда свойство (5) сохраняется для функции $g(a)$ от эрмитова элемента $a \in A$; заметим, что элемент $g(a)$ не обязан быть эрмитовым. Отправляясь от этой работы, в [19] (см. также [20], [21]) Е. А. Горин описал класс функций $g$, ограниченных в окрестности спектра $\operatorname{spec}(a) \subset \mathbb{R}$, для которых

$$
\|g(a)\|=|g(a)|_{A} .
$$


Его результат формулируется в терминах положительно определенных функций, т. е. (с точностью до нормировки) преобразований Фурье вероятностных мер на $\mathbb{R}$.

Пусть $a \in A$ - эрмитов элемент со спектром $Q=\operatorname{spec}(a) \subset \mathbb{R}$, и пусть $\lambda_{0}-$ точка максимума функции $\left.g\right|_{Q}$. Пусть функция $h: Q-\lambda_{0} \rightarrow \mathbb{R}$ определена равенством

$$
g\left(\lambda_{0}\right) h(\lambda)=g\left(\lambda+\lambda_{0}\right)
$$

Тогда (6) верно при условии, что для каждой такой точки $\lambda_{0}$ функция $h$ в некоторой окрестности множества $Q-\lambda_{0}$ является следом положительно определенной функции на $\mathbb{R}$. Если же $Q-\lambda_{0}-$ множество спектрального синтеза (например, отрезок на оси), то условие заключается просто в том, что $\left.h\right|_{Q-\lambda_{0}}-$ след положительно определенной функции для каждой точки максимума $\lambda_{0}$. Это последнее условие является также необходимым.

Выбирая подходящие $a$ и $g$, отсюда можно извлечь многие известные неравенства бернштейновского типа, в частности неравенства для дробных степеней оператора дифференцирования.

В [32] и [27], обобщая эти результаты на случай локально компактных абелевых групп (в частности, $\mathbb{R}^{n}$ ), Е. А. Горин ввел понятия символа и универсального символа. Универсальный символ - это функция на $\mathbb{R}^{n}$, применение которой к любому набору $n$ коммутирующих эрмитовых элементов сохраняет совпадение нормы и спектрального радиуса. В работе [33] Е. А. Горин и его аспирант С. Норвидас дали критерий универсальности и описали широкий класс универсальных символов. Это позволяет получать новые неравенства бернштейновского типа.

Положительно определенные функции надолго стали излюбленным горинским инструментом и объектом исследования (см. [32], [34]). Например, с их помощью Евгению Алексеевичу удалось получить в [28] аналог асимптотического закона распределения простых чисел в ситуации, когда роль целых чисел играет произвольная счетно порожденная свободная абелева группа, а роль простых чисел играют ее базисные элементы.

В начале 1980-х Е. А. Горин заинтересовался задачами обобщения теоремы единственности для потенциалов Рисса на случай произвольного банахова пространства. В работах [22], [23] Евгений Алексеевич и его ученик А. Л. Колдобский нашли метод решения подобного типа задач. Ими было введено понятие исключительного показателя банахова пространства $E: \lambda>0$ является исключительным показателем для $E$, если найдутся две различные меры $\mu_{1} \neq \mu_{2}$ на $E$ такие, что для любого $a \in E$ выполняется равенство

$$
\int_{E}\|x-a\|^{\lambda} d \mu_{1}(x)=\int_{E}\|x-a\|^{\lambda} d \mu_{2}(x)<\infty .
$$

Используя технику преобразования Фурье, удалось, например, доказать, что если $E$ есть $n$-мерное $l_{p}$-пространство с $p \geqslant 1$, то показатель $\lambda>0$ является исключительным тогда и только тогда, когда $\lambda / p$ - целое число и к тому же выполнено одно из следующих трех условий: либо $\lambda / p<n$, либо $p$ четное, либо, наконец, $p$ и $\lambda / p-n$ оба нечетны. Однако прием с преобразованием Фурье не работает в бесконечномерном случае. Поэтому потребовался новый остроумный подход к уравнениям в свертках, основанный на лемме Картана о покрытиях. В частности, для пространства $L_{p}$ исключительными оказались показатели $\lambda$, для которых $\lambda / p$ целое. В случае пространств $C(K)$ непрерывных функций на компакте для любого $\lambda>0$ имеет место единственность. Результаты этой работы нашли применения к описанию линейных изометрий одних банаховых пространств в другие (А.Л. Колдобский). Евгений Алексеевич неоднократно возвращался к этой задаче. Например, в [35] он обобщил ее результаты на случай квазинормированных пространств. 
Помимо уже упомянутых результатов Е.А. Гориным в последние десятилетия (и в последние годы) опубликован еще целый ряд других замечательных работ по различным вопросам функционального анализа: обобщение неравенства Хинчина (совм. с С. Ю. Фаворовым, [24]), о топологической характеризации мер Хаара на компактных группах [26], о регулярности групповых алгебр [29], о функциях Мёбиуса в абелевых полугруппах [30]; предложен и изучен относительный вариант теоремы Титчмарша о свертке (совм. с Д. В. Трещёвым, [31]).

Евгений Алексеевич Горин был выдающимся ученым, внесшим существенный (иногда - определяющий) вклад в развитие нескольких, казалось бы, весьма далеких друг от друга областей математики. Некоторые его идеи еще ожидают дальнейшего развития.

Евгений Алексеевич в высшей степени отличался готовностью обсуждать математические проблемы, делиться своими идеями и помогать коллегам в реализации их планов. Его сверкающее остроумие всегда было доброжелательным к собеседнику. Сочетание острого и глубокого ума, редкого обаяния, бескорыстия, доброты привлекало к нему большое количество людей. В жизни многих из нас дружба с Евгением Алексеевичем, его поддержка в трудную минуту были чрезвычайно важны.

Память о Евгении Алексеевиче будет всегда жить с нами и согревать наши сердца.

Авторы признательны за поддержку и замечания всем, кто прочел этот текст до его публикации, выразил солидарность, поделился своими воспоминаниями, разделяет с нами любовь и уважение к Евгению Алексеевичу Горину, чьи жизнь, творчество и деятельность давали реальную осмысленность и наполненность термину математическое сообщество, делая его вполне осязаемым фактом нашей жизни: М. Л. Аграновскому, Т. М. Бандман, В.Н.Бергельсону, В. М. Бухштаберу, В. А. Васильеву, А. М. Вершику, С. Г. Гиндикину, Е. И. Гордону, В. В. Грушину, В. П. Гурарию, Я. И. Житомирскому, М. И. Зеликину, В. М. Зинде, Н. М. Зобину, Ю. С. Ильяшенко, Р. С. Исмагилову, Ш. И. Калиману, А. А. Кириллову, С. В. Кислякову, В. И. Кононенко, П. А. Кучменту, М. Ю. Любичу, Ю. И. Любичу, Ю. И. Манину, Г. А. Маргулису, А. С. Маркусу, В. Д. Мильману, А. А. Миротину, А. М. Олевскому, Г. И. Ольшанскому, Б. Т. Поляку, Н. Х. Розову, В.А. Скворцову, А. М. Степину, В. М. Тихомирову, В. А. Ткаченко, Д. В. Трещёву, А. Я. Хелемскому.

Ю.А. Брудный, М.Г. Зайденберг, А.Л. Колдобский, В. Я. Лин, Б. С. Митягин, С. Норвидас, Е.М. Семенов, П.В. Семенов

\section{Список избранных работ Е. А. Горина}

[1] "О системах норм в счетно-нормированном пространстве", УМН, 13:5(83) (1958), 179-184 (совм. с Б. С. Митягиным).

[2] "О равномерно-топологическом вложении метрических пространств в евклидовы и гильбертово пространства”, УМН, 14:5(89) (1959), 129-134.

[3] "Об асимптотических свойствах многочленов и алгебраических функций от нескольких переменных", УМH, 16:1(97) (1961), 91-118; англ. пер.: "Asymptotic properties of polynomials and algebraic functions of several variables", Russian Math. Surveys, 16:1 (1961), 93-119.

[4] "О квадратичной суммируемости решений дифференциальных уравнений в частных производных с постоянными коэффициентами”, Сиб. матем. журн., 2:2 (1961), 221-232.

[5] “К определению гипоэллиптических уравнений”, УМН, 16:5(101) (1961), 163-166 (совм. с В. В. Грушиным). 
[6] "Характеристика кольца всех непрерывных функций на бикомпакте", Докл. АН CCCP, 142:4 (1962), 781-784; англ. пер.: "A property of the ring of all continuous functions on a bicompactum", Soviet Math. Dokl., 3 (1962), 159-162.

[7] "Дифференциальные уравнения, чьи решения становятся более гладкими после дифференцирования", Вестн. Моск. ун-та. Сер. 1. Матем., мех., 1963, № 2, 25-32 (совм. с В. В. Грушиным).

[8] "О разрешимости задачи Коши в классе квадратично суммируемых функций для систем дифференциальных уравнений в частных производных с постоянными коэффициентами", Вестн. Моск. ун-та. Сер. 1. Матем., мех., 1965, № 4, 6-12.

[9] "О некоторых локальных теоремах для уравнений в частных производных с постоянными коэффициентами”, Тр. ММО, 14, Изд-во Моск. ун-та, М., 1965 , 200-210 (совм. с В. В. Грушиным); англ. пер.: "Local theorem for partial differential equations with constant coefficients", Trans. Moscow Math. Soc., 14 (1965), 215-228 (with V. V. Grushin).

[10] "Максимальные подалгебры коммутативных банаховых алгебр с инволюцией", Матем. заметки, 1:2 (1967), 173-178; англ. пер.: "Maximal subalgebras of commutative Banach algebras with involution", Math. Notes, 1:2 (1967), 117-120.

[11] "Подалгебры конечной коразмерности”, Матем. заметки, 6:3 (1969), 321-328; англ. пер.: "Subalgebras of finite codimension", Math. Notes, 6:3 (1969), 649-652.

[12] "Алгебраические уравнения с непрерывными коэффициентами и некоторые вопросы алгебраической теории кос", Матем. сб., 78(120):4 (1969), 579-610 (совм. с В.Я. Лином); англ. пер.: "Algebraic equations with continuous coefficients and some problems of the algebraic theory of braids", Math. USSR-Sb., 7:4 (1969), 569-596 (with V. Ya. Lin).

[13] "Функционально-алгебраический вариант теоремы Бора-ван Кампена", Матем. сб., 82(124):2(6) (1970), 260-272; англ. пер.: "A function-algebra variant of a theorem of Bohr-van Kampen", Math. USSR-Sb., 11:2 (1970), 233-243.

[14] "Максимальные инвариантные подалгебры в алгебрах с инволюцией", Матем. cб., 85(127):3(7) (1971), 373-387 (совм. с В.М. Золотаревским); англ. пер.: "Maximal invariant subalgebras in algebras with involution", Math. USSR-Sb., 14:3 (1971), 367-382 (with V. M. Zolotarevskii).

[15] "О некоторых характеристических свойствах алгебры всех непрерывных функций на локально связном компакте", Изв. АН Арм. ССР, 11:3 (1976), 237-255 (совм. с М. И. Караханяном).

[16] "Замечание о нелокальных алгебрах", Исследования по линейным операторам u теории функиий. VII, Зап. науч. сем. ЛОМИ, 65, Изд-во "Наука", Ленинград. отд., Л., 1976, 172-177 (совм. с Б. Т. Батикяном); англ. пер.: "A remark on nonlocal algebras", J. Soviet Math., 16:3 (1981), 1167-1170 (with B. T. Batikyan).

[17] “Асимптотический вариант теоремы Фуглида-Путнама о коммутаторах для элементов банаховых алгебр”, Матем. заметки, 22:2 (1977), 179-188 (совм. с М.И. Караханяном); англ. пер.: "An asymptotic variant of the Fuglede-Putnam theorem on commutators for elements of Banach algebras", Math. Notes, 22:2 (1977), 591-596 (with M. I. Karahanyan).

[18] "Несколько замечаний в связи с теоремами Гельфанда о группе обратимых элементов банаховой алгебры", Функи. анализ и его прил., 12:1 (1978), 70-71; англ. пер.: "Some remarks in connection with Gel'fand's theorems on the group of invertible elements of a Banach algebra", Funct. Anal. Appl., 12:1 (1978), 54-55. 
[19] "Об исследованиях Г.Е. Шилова по теории коммутативных банаховых алгебр и их дальнейшем развитии", УМH, 33:4(202) (1978), 169-188; англ. пер.: "On the research of G. E. Shilov in the theory of commutative Banach algebras and their subsequent development", Russian Math. Surveys, 33:4 (1978), 193-217.

[20] "Неравенства Бернштейна с точки зрения теории операторов", к 100-летию Харьк. матем. об-ва, Вестн. Харък. ун-та. Сер. прикл. матем. и мех., 205:45 (1980), 77-105; англ. пер.: "Bernstein's inequality from the point of view of operator theory", Selecta Math. Soviet., 7:3 (1988), 191-219.

[21] Изометрические представления и дифференциальные неравенства, Ярославск. ун-т, Ярославль, 1981, 92 с. (совм. с Ю. А. Брудным).

[22] "О потенциалах, идентифицирующих меры в банаховых пространствах", Докл. AH CCCP, 285:2 (1985), 270-274 (совм. с А.Л. Колдобским); англ. пер.: "On potentials identifying measures in Banach spaces", Soviet Math. Dokl., 32 (1985), 659-663 (with A. L. Koldobskii).

[23] "О потенциалах мер в банаховых пространствах", Сиб. матем. журн., 28:1 (1987), 65-80 (совм. с А. Л. Колдобским); англ. пер.: "On potentials of measures in Banach spaces", Siberian Math. J., 28:1 (1987), 46-58 (with A. L. Koldobskii).

[24] "Обобщение неравенства Хинчина", Теория вероятн. и ее примен., 35:4 (1990), 762-767 (совм. с С. Ю. Фаворовым); англ. пер.: "Generalizations of Khinchin's inequality", Theory Probab. Appl., 35:4 (1991), 766-771 (with S. Yu. Favorov).

[25] "Обобщение одной теоремы Фугледе", Алгебра и анализ, 5:4 (1993), 83-97; англ. пер.: "An extension of a theorem of Fuglede", St. Petersburg Math. J., 5:4 (1994), $733-744$.

[26] "О топологической эквивалентности мер Хаара на компактных группах", Maтем. заметки, 68:2 (2000), 188-194; англ. пер.: "Topological equivalence of Haar measures on compact groups", Math. Notes, 68:2 (2000), 167-172.

[27] "Universal symbols on locally compact abelian groups", Bull. Polish Acad. Sci. Math., 51:2 (2003), 199-204.

[28] "Асимптотический закон распределения простых чисел в контексте свободных абелевых полугрупп”, Чебышевский сб., 6:2 (2005), 100-128; англ. пер.: "Asymptotic law for the distribution of prime numbers in the context of free Abelian semigroups", Russian J. Math. Phys., 13:1 (2006), 31-54.

[29] “О регулярности групповых алгебр", Матем. сб., 200:8 (2009), 63-78; англ. пер.: "Regularity of group algebras", Sb. Math., 200:8 (2009), 1165-1179.

[30] "Функция Мёбиуса на абелевых полугруппах", Функи. анализ и его прил., 45:1 (2011), 88-93; англ. пер.: "The Möbius function on Abelian semigroups", Funct. Anal. Appl., 45:1 (2011), 73-76.

[31] "Относительный вариант теоремы Титчмарша о свертке", Функи. анализ и его nрил., 46:1 (2012), 31-38 (совм. с Д. В. Трещёвым); англ. пер.: "Relative version of the Titchmarsh convolution theorem", Funct. Anal. Appl., 46:1 (2012), 26-32 (with D. V. Treshchev).

[32] "Положительно определенные функции как инструмент математического анализа", Фундамент. и прикл. матем., 17:7 (2012), 67-95; англ. пер.: "Positive definite functions as an instrument of mathematical analysis", J. Math. Sci. (N. Y.), 197:4 (2014), 492-511. 
[33] "Универсальные символы на локально компактных абелевых группах", Функи. анализ и его прил., 47:1 (2013), 1-16 (совм. с С. Норвидасом); англ. пер.: "Universal symbols on locally compact Abelian groups", Funct. Anal. Appl., 47:1 (2013), 1-13 (with S. Norvidas).

[34] "Некоторые неравенства для положительно определенных функций", Функи. анализ и его прил., 49:4 (2015), 76-78; англ. пер.: "Inequalities for positive-definite functions", Funct. Anal. Appl., 49:4 (2015), 301-303.

[35] "Различающие потенциалы мер в некоторых квазинормированных пространствах", Функи. анализ и его прил., 50:2 (2016), 1-19; англ. пер.: "Discriminating potentials of measures on certain quasi-normed spaces", Funct. Anal. Appl., 50:2 (2016), 83-97. 